\title{
The message writing process behind SmartAPPetite, a smartphone application, for improving food knowledge and dietary behaviours among high school adolescents
}

\author{
M. Assaff ${ }^{1}$, C. O'Connor ${ }^{1}$, J. Siu' ${ }^{1}$, N. Woods², R. Brown², A. Clark², S. Rinaldi², A. Piaskoski², S. \\ Doherty $^{3}$, J. Haines ${ }^{4}$, L. Minaker ${ }^{3}$, K. Norozi ${ }^{5}$, J. Seabrook ${ }^{1}$, P. Wilk ${ }^{5}$, S. Stranges ${ }^{5}$, J. Gilliland ${ }^{2}$ \\ ${ }^{1}$ Brescia University College, London, ON, ${ }^{2}$ Human Environments Analysis Laboratory, Western University, London, ON, ${ }^{3}$ Wilfrid Laurier \\ University, Waterloo, ON, ${ }^{4}$ University of Guelph, Guelph, ON, ${ }^{5}$ Western University, London, ON
}

\section{Introduction:}

Only $30 \%$ of Canadian adolescents consume a diet rich in vegetables and fruits. Poor dietary behaviours in adolescence can carry into adulthood and contribute to the development of chronic disease. Since the majority of adolescents own smartphones, smartphone-based interventions to improve food knowledge and dietary behaviours are a logical approach.

\section{Purpose:}

To describe the message-writing process developed to ensure consistent, evidence-based nutrition messages for a smartphone application, SmartAPPetite.

\section{What is SmartAPPetite?}

SmartAPPetite is a multidimensional smartphone application that sends nutrition and lifestyle messages to help its users make healthier choices. It was developed through an interdisciplinary collaboration with an overall goal of improving food knowledge, food purchasing, and diet quality of adolescents.
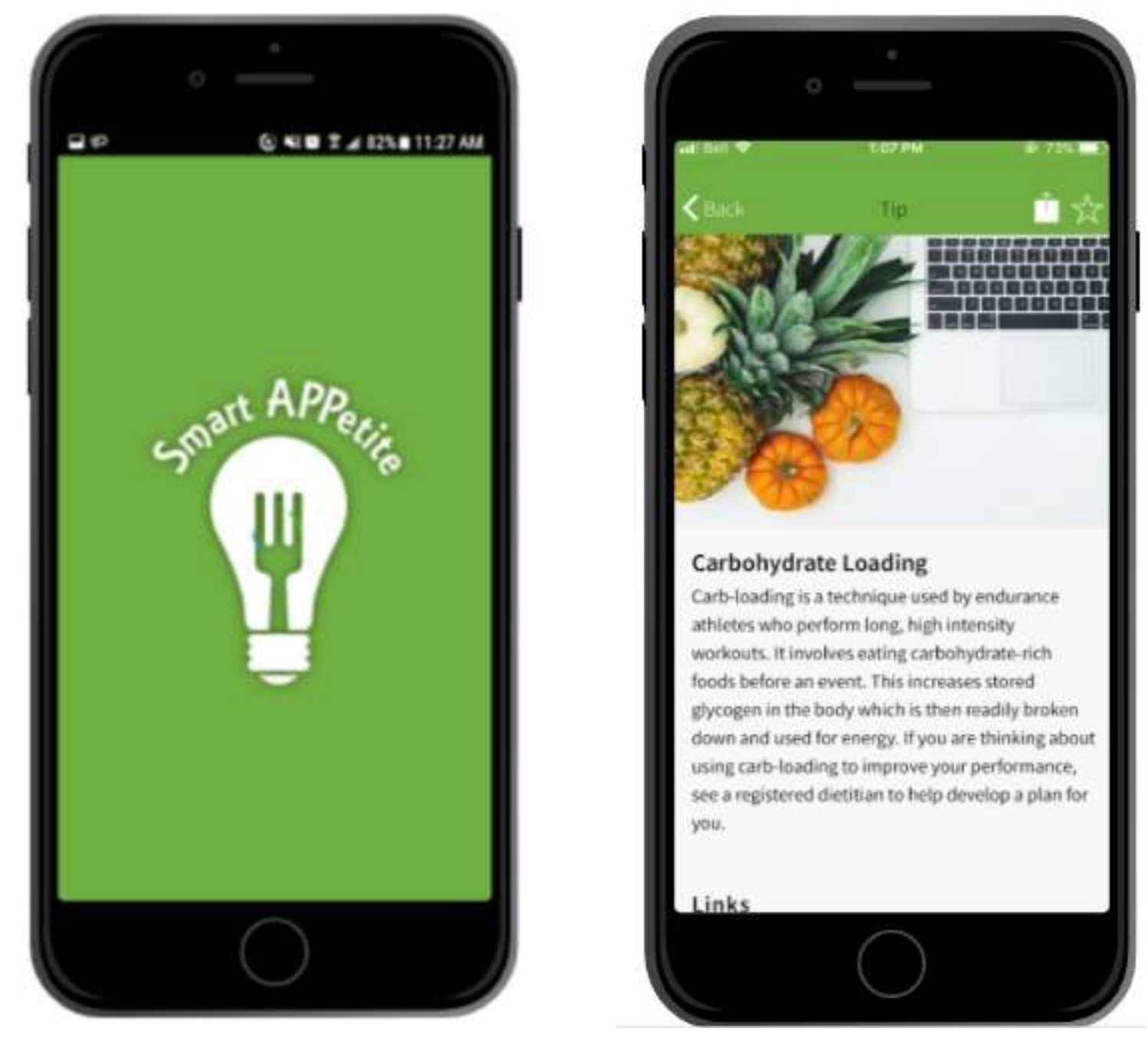

\section{Systematic Approach Used:}

A database of over 1000 messages was created with a range of nutrition and lifestyle topics, such as sports nutrition, eating away from home, information about specific nutrients, seasonality and origin of foods, and how to choose, prepare, and store fresh food items. A writing guide was created and used to standardize the messages which included dietitian approved sources to gather nutrition information. A Youth Advisory Council of high-school students assisted with the selection of topics and assessing the relatability of messages. Using program algorithms, SmartAPPetite selected messages from the database according to the users age, sex, and reported dietary behaviours. User feedback also allowed the app to continually adjust message selection algorithms.

SmartAPPetite message-writing process:

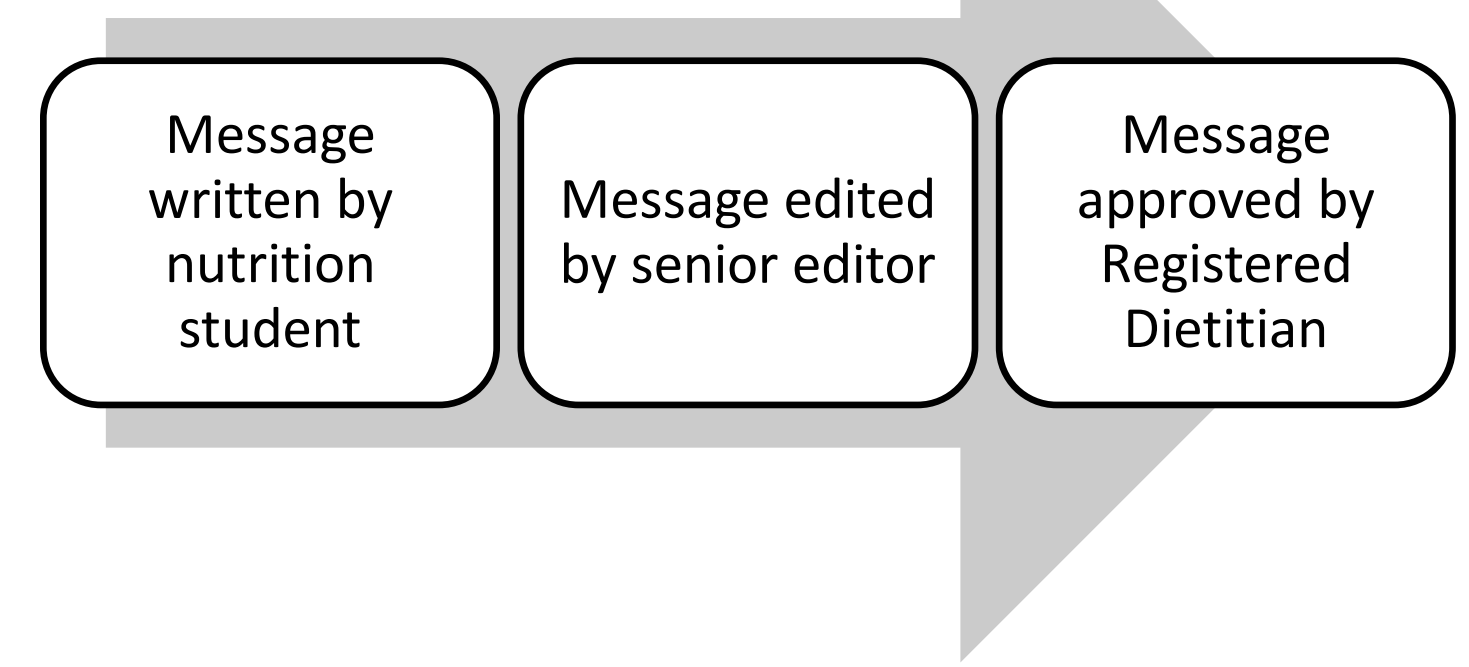

\section{Conclusions:}

SmartAPPetite messages have undergone a thorough planning, writing, editing, and approval process to ensure users are provided with evidence-based and expert recommended nutrition and lifestyle messages.

\section{Significance to field of dietetics:}

Nutrition-related smartphone applications have the potential to reach a large proportion of Canadian adolescents and enhance dietary behaviours. 